Supporting information:

\title{
Effect of Electrostatic Interaction on the Photophysical Properties of the Composites of CdTe Nanocrystals and Carbazole-Containing Polymers
}

\author{
Haizhu Sun, ${ }^{\dagger,}{ }^{\ddagger}$ Hao Zhang, ${ }^{\dagger}$ Junhu Zhang, ${ }^{\dagger}$ Yang Ning, ${ }^{\dagger}$ Tongjie Yao, ${ }^{\dagger}$ Xin
}

Bao, ${ }^{\ddagger}$ Chunlei Wang, ${ }^{\dagger}$ Minjie Li, ${ }^{\dagger}$ and Bai Yang ${ }^{*}{ }^{\dagger}$

${ }^{\dagger}$ State Key Lab for Supramolecular Structure \& Materials, College of Chemistry, Jilin University, Changchun 130012 (P. R. China)

* College of Chemistry, Northeast Normal University, Changchun 130024 (P. R. China)

E-mail: byangchem@jlu.edu.cn

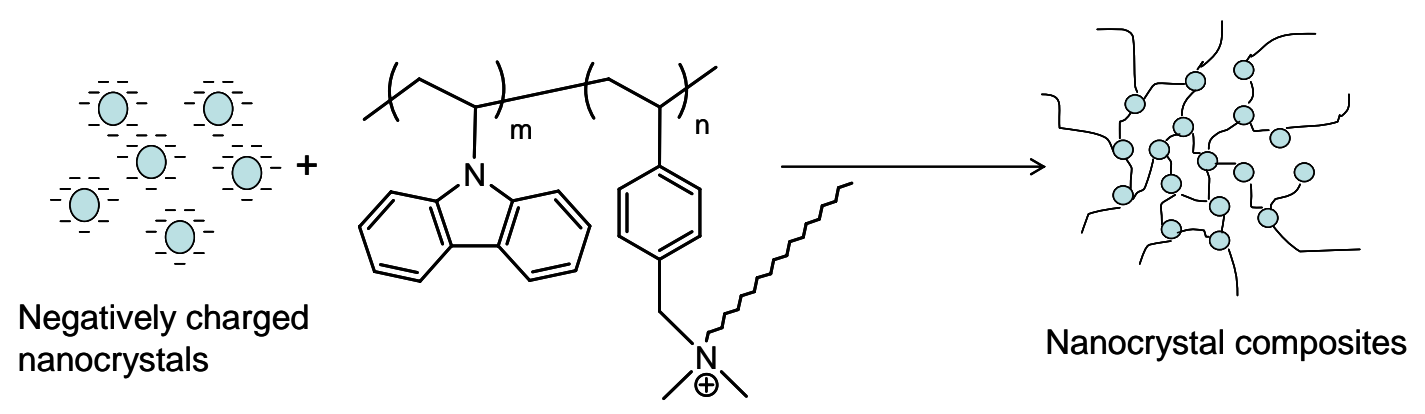

Scheme S1 Summary of the transfer process of CdTe NCs from aqueous phase to organic phase 

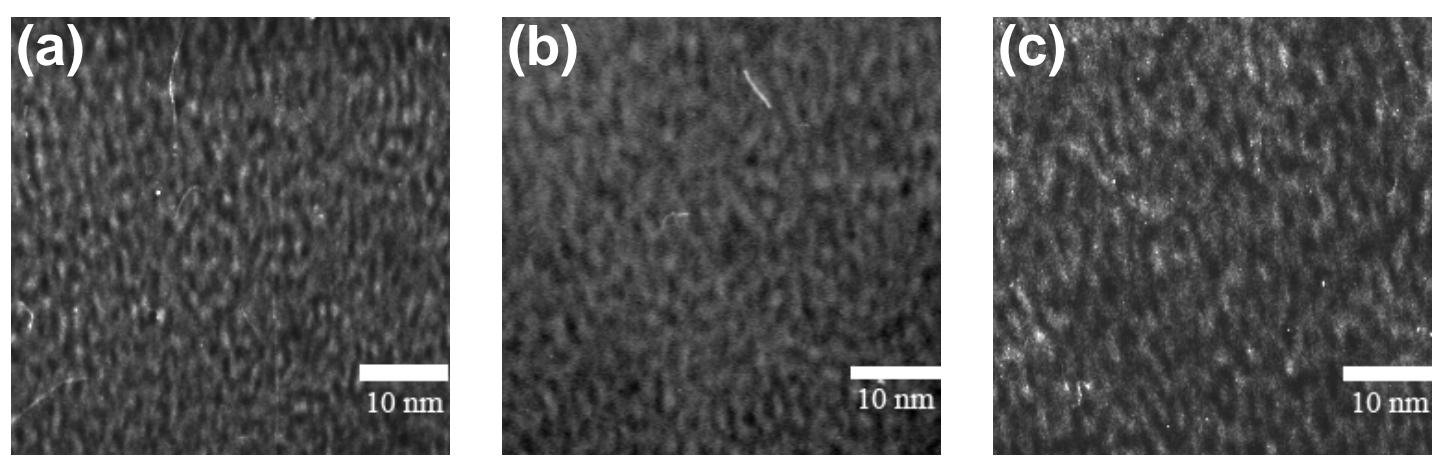

Figure S1. TEM images of the composites with CdTe NCs content: (a) 5.4 wt.-\%, (b)

12.8 wt. -\%, and (c) 28.6 wt. - \%.
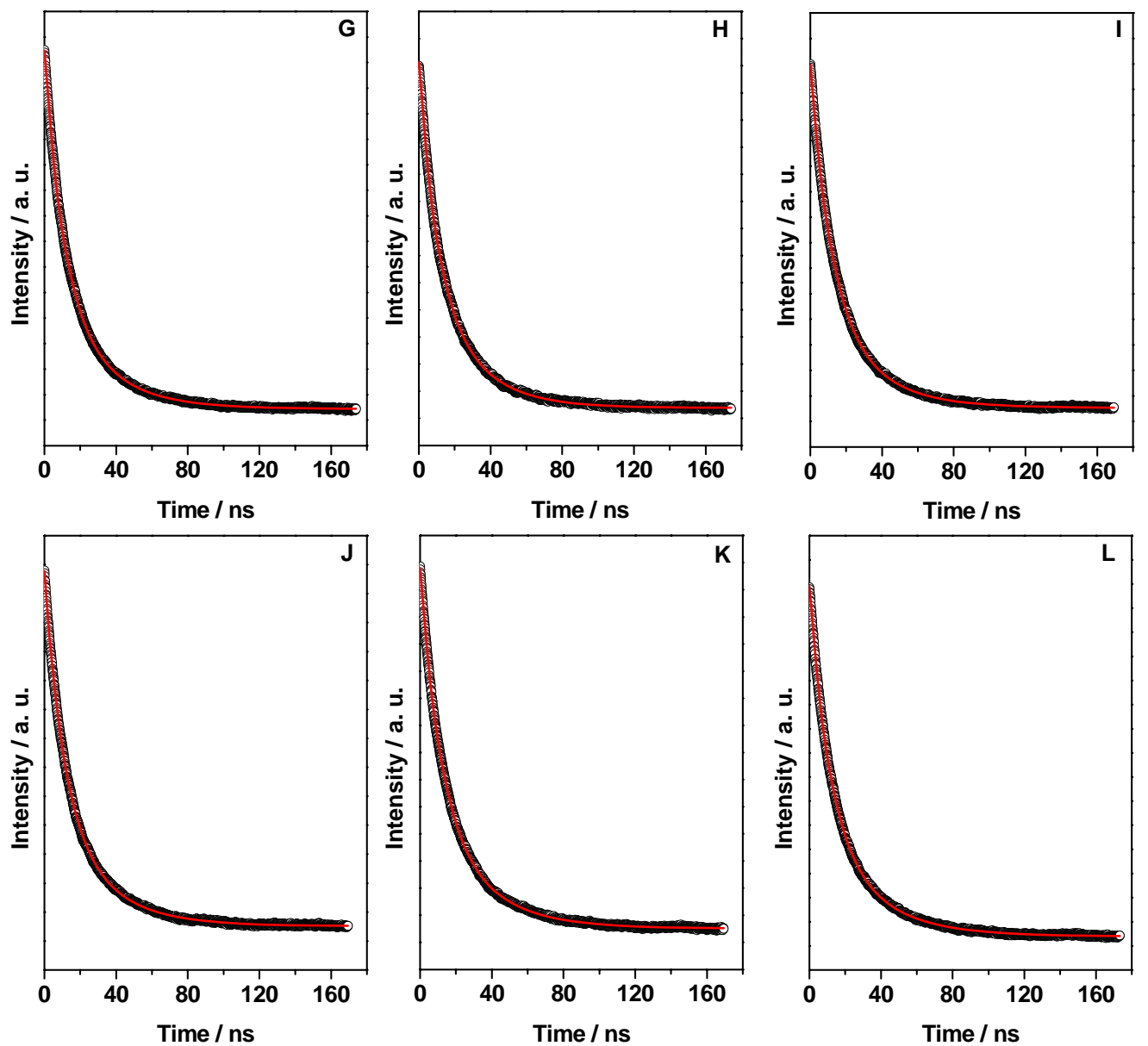

Figure S2. Lifetime decay of composite G-L. Black circles: experiment data. Red line: Fitted by biexponential function $A_{1} \exp \left(\frac{t}{\tau_{1}}\right)+A_{2} \exp \left(\frac{t}{\tau_{2}}\right)$. 

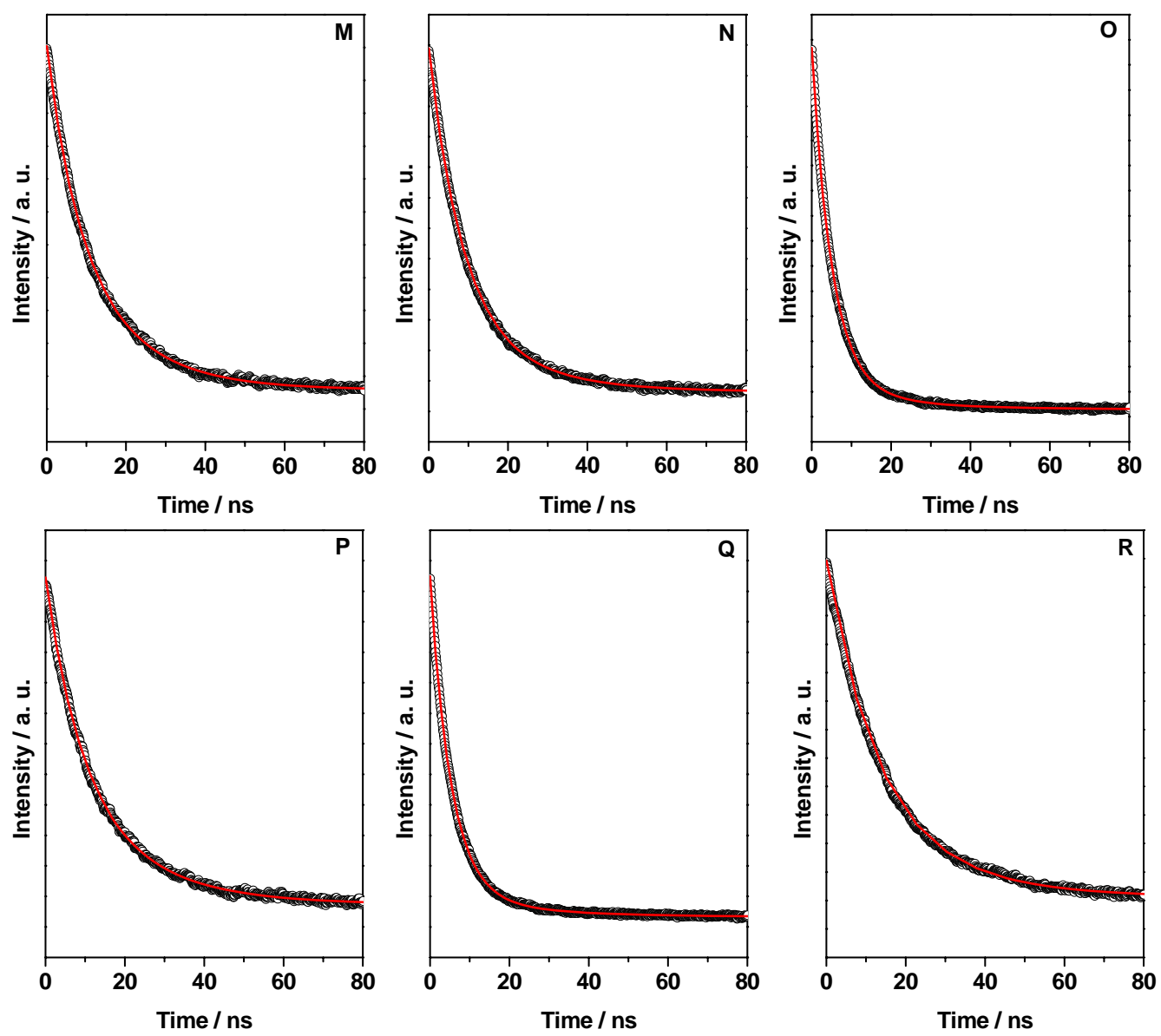

Figure S3. Lifetime decay of composite M-R. Black circles: experiment data. Red line: Fitted by biexponential function $A_{1} \exp \left(\frac{t}{\tau_{1}}\right)+A_{2} \exp \left(\frac{t}{\tau_{2}}\right)$. 

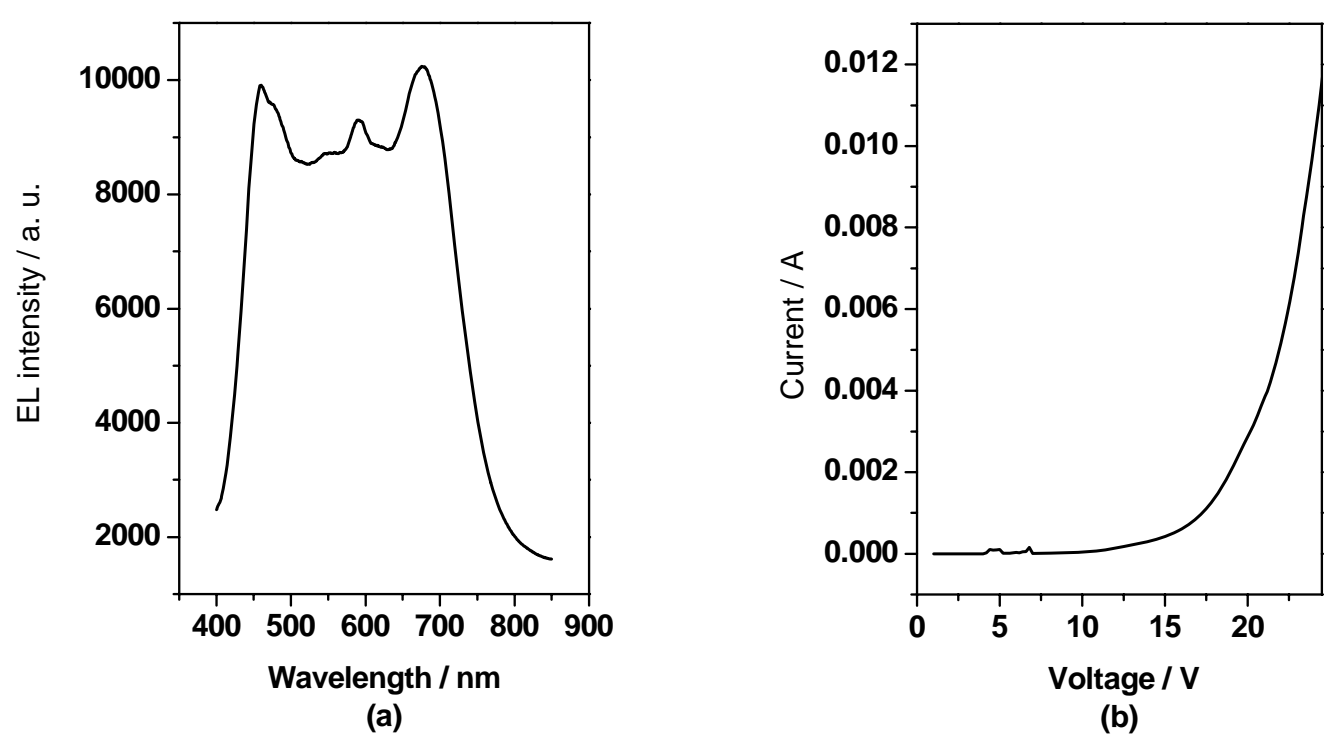

Figure S4 (a) EL spectra of CdTe NC-CPVKOVDAC composite with white-light emission. (b) $I-V$ characteristic of the LED device.

For preparation of composite with white-light emission, polymer solution (3 mL) with the concentration $10 \mathrm{mg} / \mathrm{mL}$ was added to CdTe NCs aqueous solution (12 mL) containing two types of CdTe NCs with green and red emission in a ratio of 1:4 (green to red). The solvent was removed under reduced pressure and the resulting composite solids were kept in vacuum overnight. The structure of the fabricated devices in this study is indium tin oxide (ITO)/ 3, 4-polyethylenedioxythiophene-polystyrene sulfonate (PEDOT: PSS) (35 nm) /composite: 2-(4-biphenylyl)-5-(4-tert-butylphenyl)-1, 3, 4-oxdiazole (PBD) (100-130 nm)/Al (150 nm). The composite $(5 \mathrm{mg} / \mathrm{mL})$ solution was spin cast $(2500 \mathrm{r} / \mathrm{min})$ on the top of PEDOT: PSS layer to form an emissive layer. An aluminum electrode was vacuum evaporated through a mask on top of the film. Current-brightness-voltage characteristics were measured by using a Keithley source measurement unit (Keithley 
2400 and Keithley 2000) with a calibrated silicon photodiode. Electroluminescence was detected by JY SPEX CCD 3000 spectrometer. All the measurements were carried out in ambient atmosphere at room temperature. The active area of device was $16 \mathrm{~mm}^{2}$. 